\title{
WHICH IS YOUR DIAGNOSIS?
}

Male patient, 18 years old, complaining of pain in his left knee for four months, with no history of trauma.

\section{Imaging findings}

Figure 1: Tibia proximal, epiphyseal, rounded lytic lesion presenting well defined and regular margins, without surrounding sclerosis and with central gross calcification.

Figure 2: Tibia proximal, epiphyseal, rounded lytic lesion presenting well defined and regular margins, without surrounding sclerosis and with central gross calcification. Cortical rupture and articular extension are observed.

Figure 3: Low signal on proximal, epiphyseal, rounded lytic image presenting well defined margins and calcifications inside.

Figure 4: Lesion presenting an intense surrounding bone-marrow edema and high signal intensity inside, with central calcification presenting low-signal.

Figure 5: Lesion presenting an intense surrounding bone-marrow edema and high signal intensity, with central calcification presenting low-signal. Presence of thickening and adjacent periosteal hypersignal.

Figure 6: Enhancement inside the lesion (solid-matrix). Bone-marrow edema also presents significant enhancement. Additionally, slight synovial enhancement is observed, indicating synovitis.

Diagnosis: Chondroblastoma.

\section{COMMENTS}

Chondroblastoma is a rare cartilaginous neoplasm that characteristically arises in the epiphysis of long bones, principally in young patients, and accounting for less than $1 \%$ of all primary bone tumors ${ }^{(\mathbf{1 , 2})}$.

These tumors affect principally people between 10 and 30 years of age, however, Chondroblastoma has been reported to arise in individuals in the age range between 3 and 73 years ( $90 \%$ occur between 5 and 25 years of age).

The most common site is the lower extremity ( $72 \%$ of cases), and $50 \%$ of tumors occur around the knee. Studies show similar figures in cases localized in the distal femur, proximal tibia and proximal humerus (20\% each). The remainder originates from flat bones and small tubular bones of hands and feet ${ }^{(2)}$. 
The clinical picture is quite non-specific and includes local pain, tenderness, swelling, and withered or shrunken appearance of the muscle near the affected bone muscle. Articular effusion may be present.

Chondroblastomas generally arise or are centered in the epiphysis (more than $95 \%$ of cases). However, as the lesion develops, it may extend to the adjacent metaphysis with endosteal irregularities, adjacent cortical bone remodeling and periosteal reaction. Periosteal reaction occurs in $15-30 \%$ of cases and may be solid or lamellated ("onion-skin"), but never in an aggressive way, for example in a pattern sometimes called "sunburst" or "hair-on-end" or Codman's triangle ${ }^{(3)}$.

For imaging assessment of chondroblastomas, X-ray demonstrates a radiolucent lesion $40 \%$ are uniformly radiolucent and $60 \%$ present as mottled opacity because of chondroid matrix, amorphous calcifications and peripheral septa -, oval in shape, geographic, well defined margins, eccentrically localized in long bone epiphysis, mainly in distal femur, proximal tibia and proximal humerus.

Such lesions measure between $1 \mathrm{~cm}$ and $4 \mathrm{~cm}$ in diameter, although there are reports on lesions measuring $13 \mathrm{~cm}^{(\mathbf{1}, 2,4)}$.

Computed tomography (CT) is a better method to demonstrate the tumoral matrix, cortical destruction and possible tumor extension to soft tissues and is quite useful in the evaluation of aggressive and recurrent lesions.

On the other hand, magnetic resonance imaging (MRI) provides significant information on tumor localization, extension to metaphysis and to adjacent soft tissues. Chondroblastomas present hyposignal in T1-weighted images and variable signal in T2-weighted images, usually with peripheral ring of marked T2-hyposignal in all the sequences. Central hyposignal is observed in $\mathrm{T} 2$-weighted images resulting from the predominance of a less prominent chondroid matrix, chondroblasts, calcifications and hemosiderin deposits in the lesion. Similarly to CT, RMI can show fluid-fluid level.

The majority of chondroblastomas is associated with significant alterations in signal of bone marrow and adjacent soft tissues which besides being edematous, present an intense and homogeneous paramagnetic contrast-enhancement ${ }^{(1)}$. Such enhancement is typically larger than the enhancement of the tumor itself and some authors have observed that this finding also occurs in other benign tumors with aggressive features like osteoid osteoma and Largerhans' cells histiocytosis.

Chondroblastoma diagnosis is made by means of surgical biopsy and treatment recommended is extended surgical curettage and packing with a bone graft or polymethylmethacrylate. Approximately $25 \%$ of lesions are likely to recur, the probability of 
recurrence being higher in patients with open physis, lesions larger than $3.7 \mathrm{~cm}$ and lesions localized in the proximal femur or pelvis

Among differential diagnoses, the following entities should be considered: chondrosarcoma, eosinophilic granuloma, giant celll tumor and Largerhans'cells histiocytosis ${ }^{(1)}$.

\section{REFERENCES}

1. Ecklund K, Jaramillo D, Buonomo C. Pediatric case of the day. Chondroblastoma. RadioGraphics 1996;16:979-982.

2. Bloem JL, Mulder JD. Chondroblastoma: a clinical and radiological study of 104 cases. Skeletal Radiol 1985;14:1-9.

3. Jee WH, Park YK, McCauley TR, et al. Chondroblastoma: MR characteristics with pathologic correlation. J Comput Assist Tomogr 1999;23:721-726.

4. Gardner DJ, Azouz EM. Solitary lucent epiphyseal lesions in children. Skeletal Radiol 1988;17:497-504. 


\section{QUAL O SEU DIAGNÓSTICO?}

Figuras

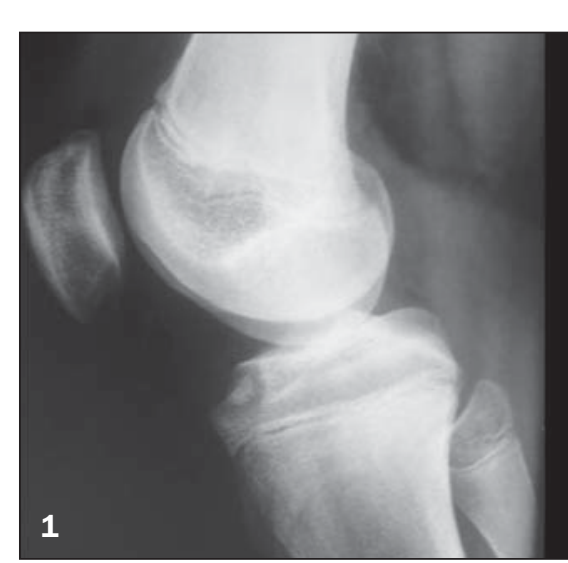

Figure 1. Profile plain X-ray of left knee.

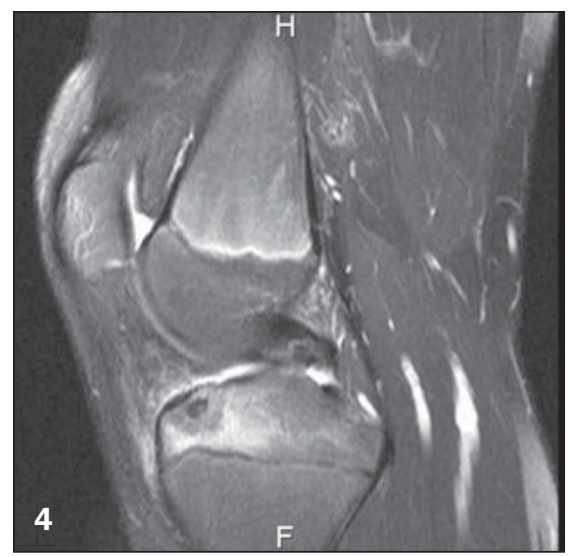

Figure 4. Magnetic resonance imaging of left knee, predominantly T2-weighted, fat-saturated sagittal image.

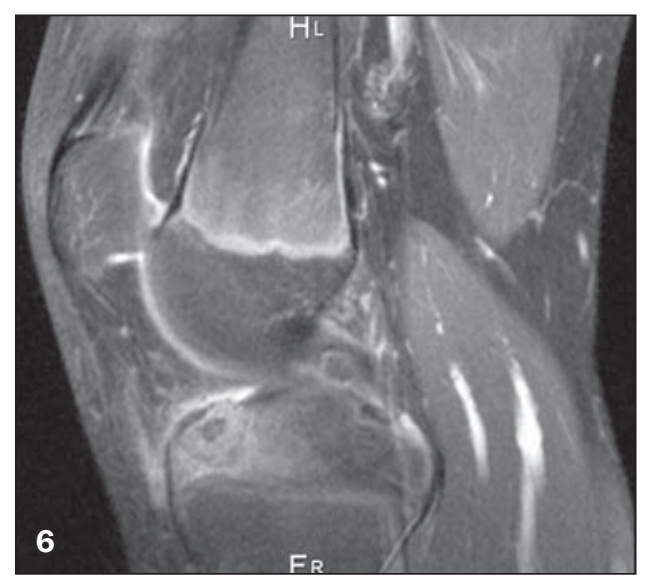

Figure 6. Magnetic resonance imaging of left knee, predominantly T1-weighted, fat-saturated, sagittal image acquired after paramagnetic contrast (gadolinium) intravenous administration.

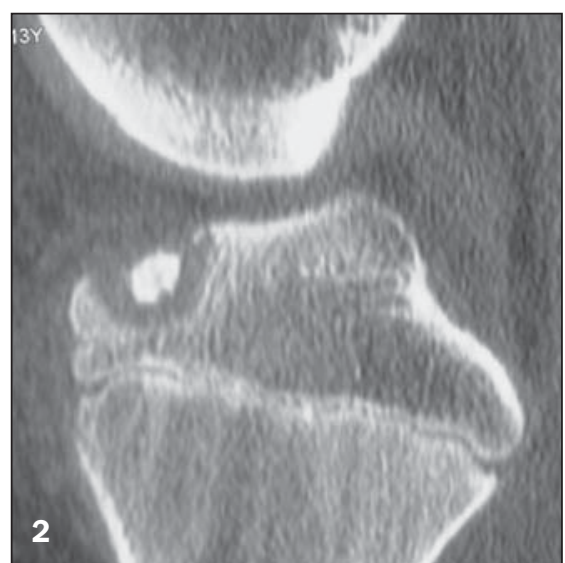

Figure 2. Computed tomography image of left knee - sagittal reconstruction.

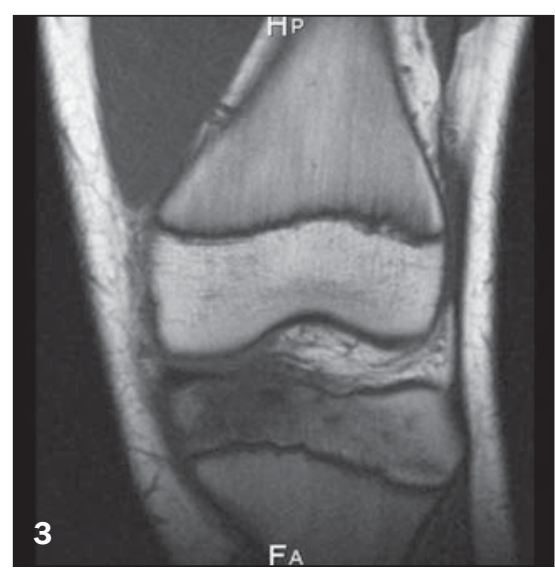

Figure 3. Magnetic resonance imaging of left knee, predominantly T1-weighted non-fat-saturated coronal image.

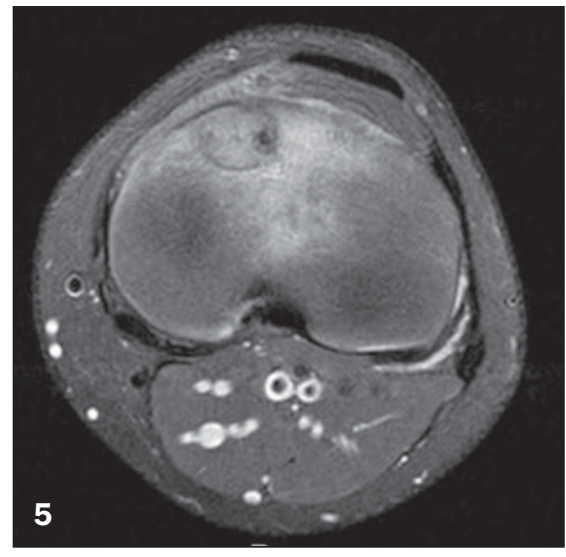

Figure 5. Magnetic resonance imaging of left knee, predominantly T2-weighted, axial, fat-saturated image.

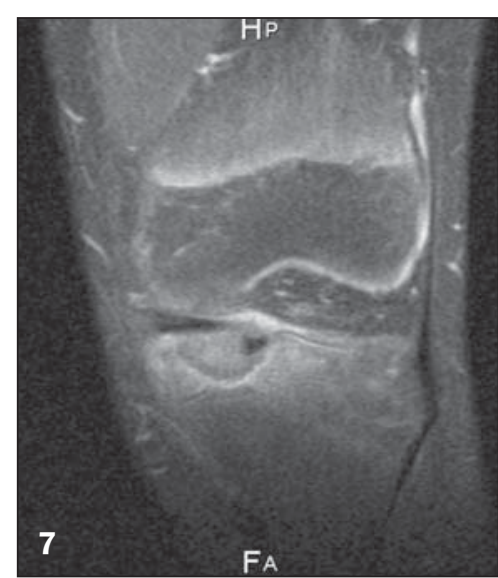

Figure 7. Magnetic resonance imaging of left knee, predominantly T1-weighted, fat-saturated, coronal image acquired after paramagnetic contrast (gadolinium) intravenous administration. 